\title{
Adherence to Antihypertensive Medications and Its Determinants in Hypertensive Patients - A Complete Review
}

\author{
Satish $\mathbf{S}^{\mathbf{1}}$, Manju Jose ${ }^{2}$, A R Shabaraya ${ }^{3}$ \\ ${ }^{1}$ Associate Professor, Department of Pharmacy Practice, ${ }^{2}$ Student, Pharm D, Department of Pharmacy Practice, \\ ${ }^{3}$ Professor \& Head, Department of Pharmacy Practice, \\ Srinivas College of Pharmacy, Mangalore, Karnataka-574143
}

Corresponding Author: Manju Jose

\begin{abstract}
Hypertension is a global health problem; it causes complications of cardiovascular diseases, stroke, and renal failure leading to early mortality and disability. Adherence to antihypertensive medications helps to control blood pressure levels. WHO defines adherence as "the extent to which a person's behavior taking medication, following a diet, and / or executing lifestyle changes-corresponds with agreed recommendations from a health care provider. Poor adherence is the major cause of uncontrolled BP. Common barriers to adherence are under the patient's control, so that attention to them is a necessary and important step in improving adherence. The factors driving patients' adherence to medication are multifactorial, but can be grouped under five main domains including socioeconomic factors, healthcare system related factors, disease related factors, therapy-related factors and patientrelated factors. Identifying factors that affect medication adherence is the first step towards improving adherence. This article covers various factors influencing medication non adherence among hypertensive patients.
\end{abstract}

Keywords: Hypertension, medication adherence

\section{INTRODUCTION}

Hypertension contributes to the burden of cardiovascular diseases, stroke, and renal failure leading to early mortality and disability. [1] High prevalence of hypertension is reported from various regions of India. Current studies have reported that hypertension is present in 25$30 \%$ urban and $10-15 \%$ rural population in India. [2] Adherence to diet, lifestyle modifications, and anti-hypertensive pharmacotherapy are pivotal in achieving optimal blood pressure (BP) control, especially for patients whose BP cannot be controlled by diet and lifestyle modification alone. ${ }^{[3]}$

WHO identifies non-adherence as the most important cause of uncontrolled blood pressure and confirm that $50-70 \%$ of population do not take their antihypertensive medication as prescribed. A study found that, patients with poor antihypertensive adherence had higher Sokolow-Lyon ECG (electro cardiograph) score, as well as prolonged $\mathrm{P}$ wave duration on ECG and higher rate of ECG LVH (left ventricular hypertrophy) compared to patients with good drug adherence. Identifying factors that affect medication adherence is the first step towards improving adherence. ${ }^{[1]}$

Non-adherence has important influence on therapeutic outcomes and leads to disease progression, increased visits to outpatient department, re-admission, and hospitalization, additional financial burden to the community by increasing the cost of treatment and hospitalization. Due to patients' non-adherence with therapy. diagnosis may become difficult for physicians; it adds to the complexity of treatment. [4] This can lead to dose 
augmentation, increasing the risk for ADRs or drug interactions. ${ }^{5]}$ According to WHO, the factors driving patients' adherence to medication are multi-factorial. [6]

\section{DEMOGRAPHIC FACTORS:}

AGE: Majority of studies showed that comprehension of drug information and adherence is lower in adults $>75$ years of age than those aged 66-74 years. The main reasons for medication non adherence in elderly patients are;

1. Potential side effects

2. Cost

3. Regimen complexity

4. Cognitive decline ${ }^{[7]}$

Studies assessed the relation between cognitive impairment and treatment adherence in elderly hypertensive patients concludes that Cognitive impairment was strongly correlated with the total score of the HBCS (Hill bone High blood pressure Compliance Scale questionnaire $(p<0.001)$ and two of its subscales: "appointment keeping" and "medication taking". [8] Medication adherence of elderly patients was associated with their education level, health-related problems, dosing frequency, satisfaction with patient counselling. ${ }^{[9]}$ Poor compliance with prescribed medications was associated with subjects aged 80 years and over, who were administering their own medication and who were not receiving pharmacist counselling. ${ }^{[10]}$

MARITAL STATUS: Being married was associated with higher probability of medication adherence. ${ }^{[11]}$ Jia-RongWu et al conducted a study on 136 subjects with heart failure and their Medication adherence was monitored objectively for 3 months using the Medication Event Monitoring System. Results indicate Unmarried patients were more likely to be non-adherent. The study suggests that these patients often require assistance to adhere to prescribed medication. ${ }^{[12]}$
EDUCATION AND GENDER: Women are significantly more likely than men to use one or more medications and women use more unique medications, on average, than men, for all clinical metrics assessed, women were less likely than men to be adherent in their use of chronic medications, and they were less likely to receive the medication and monitoring recommended by therapeutic guidelines. [13] Genderperspective on NA are scarce another reason is that women could be more sensitive to drugs compared to men due to pharmacokinetic differences. ${ }^{[14]}$

The relationship of education to medication adherence among AfricanAmerican patients varied significantly by sex. Specifically, lower educational attainment was related to higher adherence among men, but lower adherence among women. ${ }^{[15]}$ Post graduate level of education showed absolute adherence to drugs. [16] Lower medication literacy was found to be associated with inappropriate medicationtaking behavior. ${ }^{[17]}$

Knowledge of medications, especially among high literate patients greatly influenced non-adherence. Such patients were of the view that before taking the medication, they researched online and read the drug information leaflet to understand what the prescribed medication entail. Some patients claimed that their research informed awareness that taking the medications could trigger other conditions; hence they discontinued in order to avoid such episodes. ${ }^{[18]}$

\section{PSYCHOSOCIAL}

FACTORS:

Associations are found between certain psychosocial factors and adherence behavior. Generally, non-adherence can be classified as either intentional or unintentional. Intentional non-adherence can be measured in the theories of reasoned action and planned behavior, where patient's beliefs about certain behaviors are strong detectors of behavioral intention. Therefore, holding positive beliefs about medications is a prerequisite for intentional 
adherence. The impact of beliefs on adherence varies according to the culture. [19]

An international study found that Asian cultural background reported more negative views about medication than those who reported a European cultural background. Students with an Asian cultural background were significantly more likely to perceive medicines as being intrinsically harmful, addictive substances that should be avoided. [20]

Non-adherence is associated with spiritual or divine healing in the form of prayers, fasting and paranormal approaches. The belief was that prayers work better than medication because ill health is an act of God. The belief that spiritual healers have the power to heal disease conditions influenced discontinuation of the medication-taking. Prevailing norms of the cultural and social environment were shown to influence patients' discontinuation of the medication. Patients are discouraged by relatives and family members who insisted it is against societal norm to resort to modern medicine to treat illness. ${ }^{[18]}$

People who have social support from family, friends, or caregivers to assist with medication regimens have better adherence to treatment. Adherence to drug treatment was found to increase positively as the social support of patients with hypertension. [21] Assistance and support from families, friends, and other individuals have been connected with promoting patients' adherence by encouraging optimism and self-esteem, buffering the stresses of being ill, reducing patient depression, and improving sick-role behavior. ${ }^{[22]}$

\section{PATIENT-PRESCRIBER}

RELATIONSHIP: The relationship with the prescriber emerged as the most important factor for adherence to medication. Although physicians discussed the effect and dose of the medication, they spent little time discussing the effects and long-term goals of antihypertensive therapy. The patient interviews revealed that the patients had a fragmented understanding of their antihypertensive medication; 39\% reported that they did not know the effects of their medication. These findings highlighted the need of patient education on the importance of antihypertensive regimens to increase compliance. ${ }^{[23]}$

Physicians causes patients' nonadherence by prescribing complex treatments. ${ }^{[24]}$ Physicians' communication style can strongly influence patients' involvement in and outcomes of the treatment process. ${ }^{[25]}$ It is difficult to establish a relationship as patients consult different doctors each time. ${ }^{[2]}$

Concerns were raised about ambiguous and confusing explanations offered by health providers in regard to how the medications should be taken. Also provider's handwriting of the dosage was not decipherable enough. This leads to administration of dosage regimen above the recommended dosage, leading to complications. To avoid further worsening of the condition, patients had to discontinue with the medication. ${ }^{[18]}$

COST OF TREATMENT: As generic medications available at reasonable prices, nearly $30 \%$ of Americans don't take their prescribed medications because of treatment expenses. $60 \%$ of adult patients received a prescription in 2017, but due to high prescription drug costs nearly $11 \%$ didn't take their medications. ${ }^{[26]}$ People who lived far away from the hospitals tend to avoid journey due to transportation costs because of their low income. ${ }^{[2]} 25 \%$ of patients are paying more for their medications than ever before, and $14 \%$ can't fill prescriptions due to high costs. Medication subsidies may help improve medication adherence. Patients receive Medicare subsidies were more likely to take their medications than those without the subsidies. Sixty-nine percent of black patients, $70 \%$ of Hispanic patients, and $61 \%$ of white patients receiving the subsidy achieved full medication adherence by study's end. ${ }^{[27]}$ 


\section{PHYSICAL DIFFICULTIES AND FORGETFULNESS: \\ Physical}

impairments and cognitive limitations may increase the risk for non-adherence in older adults. Forgetfulness is one of the major non-intentional reasons for non-adherence. [21]

Recent research on medication adherence among disabled people is limited, but evidence has consistently shown lowadherence levels. Disabled people were especially vulnerable to various diseases related to hypertension because of a low antihypertensive adherence rate. ${ }^{[28]}$

Lower adherence is seen among people with organ impairments; disabled people have a higher susceptibility to disease and are more likely to suffer concurrently from multiple diseases. Medication adherence therefore plays a significant role in the health and survival of people with disabilities. Although low adherence has been reported among disabled people, the reasons for this have not previously have been explored. ${ }^{\text {[29] }}$

Forgetfulness caused by routine schedules compromised medication schedules. Patients who had started the treatment as directed later failed to take doses because of preoccupation with routine work, daily activities and other engagements. Patients who travelled from home frequently are more exposed to forgetfulness, and therefore non-adherence. [18]

ALCOHOL AND SMOKING: Alcohol and substance misuse being an important factor in non-adherence with medical treatment. Several studies have highlighted the direct impact of alcohol and smoking on medical conditions themselves. Most studies reported negative effects of alcohol consumption on adherence. ${ }^{\text {[30] }}$

Chris L. Bryson et al conducted a study on Alcohol Screening Scores and Medication Non-adherence founds that the percentage of adherent patients was lower in the 3 highest alcohol misuse groups. Alcohol misuse, as measured by a brief screening questionnaire, was associated with increased risk for medication non adherence. ${ }^{[31]}$ Substance use coping, in addition to other life stressors that can accompany substance abuse, might contribute to greater degrees of dysregulation, resulting in adherence difficulties. ${ }^{[32]}$

ABSENCE OF SYMPTOMS: Hypertensive patients can be asymptomatic. Majority of the patients are unaware of the asymptomatic nature of hypertension and the rationale for its treatment. ${ }^{[2]}$ Shortage of sufficient knowledge about the nature of the disease, symptoms, complications and treatment methods as well as wrong beliefs toward them are the significant factors that account for non-adherence to treatment. The causes of non-adherence to treatment in 16 countries showed that ignorance of the causes and symptoms can lead to intentional discontinuation of treatment by patients. ${ }^{[33]}$

POLYPHARMACY: Inappropriate drug combinations, unnecessary medications, and inappropriate drugs for specific patients (such as the elderly) constitute the problems of polypharmacy. Thus, patients receiving only two medications could have polypharmacy. Polypharmacy has been shown to result in Unnecessary and/or inappropriate medication prescribing, increased risk for drug interactions and ADRs, Non adherence, and increased overall drug expenditures. ${ }^{[34]}$ Polypharmacy can increase the risk for medication nonadherence, which consecutively can cause suboptimal therapeutic effectiveness and poor clinical response. ${ }^{[5]}$

Polypharmacy with age is common to address specific symptoms, improve or extend quantity of life, or heal curable conditions. Almost $20 \%$ of geriatric population (65 years or older) take 10 or more medications. Multiple medication use creates and contributes to adherence challenges in the aging population. ${ }^{[7]}$

A study performed in patients with polypharmacy reported very high 
medication adherence rates regardless of number of medicines prescribed. Therefore, physicians should not feel deterred from prescribing multiple agents in order to achieve adequate control of hyperglycemia, hypertension, and hyperlipidemia. Most patients with hypertension have positive beliefs about the necessity of their medication. ${ }^{[34]}$

Increasing number of hypertensive medications are being utilized in older Americans. High adherence to CV medications was recently shown to significantly improve healthcare outcomes and reduce annual costs for secondary prevention of CAD. Most of the expenses are incurred through inpatient care, emergency department visits, and hospitalizations as a result of complications and adverse drug events. Therefore, the growing share of US older adults with hypertension makes polypharmacy an increasingly relevant societal challenge. ${ }^{[35]}$

NATURAL REMEDIES: It is confirmed that up to $80 \%$ of the world's population rely on herbal medicinal products as a primary source of healthcare and traditional medical practice. The main reasons for the utilization of natural remedies include patients being uncomfortable about discussing their medical problems, fear of possible misdiagnosis and wrong treatment, lack of time to see a physician. Many patients select herbal medicines from an approach based on anecdotal information, that is, "it worked for my friend or relative". The general perception that herbal remedies are very safe but herbs have been shown to be capable of producing a wide range of undesirable or adverse reactions some of which are capable of causing serious injuries, life-threatening conditions, and even death. ${ }^{[36]}$

\section{ADVERESE EFFECTS OF}

MEDICATIONS: A study conducted in Swedish general population claims that the patient's attitude toward drugs in general can greatly influence adherence. These attitudes, which reflect evaluation of the object (i.e. the drug) as good or bad, harmful or beneficial, are thought to influence behaviour and, consequently, adherence. A belief that medication is harmful has been associated with decreased adherence, and this study confirmed that a negative attitude toward drugs seemed to be connected with NA. ${ }^{[37]}$

A recent study concludes that the drug's side effect contributes to antihypertensive medication non-adherence among hypertensive patients. Among the study participants, $21.3 \%$ think that their symptoms were caused by their antihypertensive medications and they were more likely to have poor adherence than patients who do not know. ${ }^{[14]}$ Another study demonstrated that $85 \%$ of the participants experienced side effects mainly excessive urination and decrease in sexual drive and $34.5 \%$ became non-adherent. ${ }^{[38]}$

DURATION OF TREATMENT: A longitudinal study of electronically compiled dosing histories of 4783 patients confirm that, half of the patients prescribed an antihypertensive drug stopping taking it within 1 year of treatment. Despite the availability of effective treatment, over half of the patients being treated for hypertension drop out of care entirely within a year of diagnosis and of those who remain under medical supervision only about $50 \%$ take at least $80 \%$ of their prescribed medications. Consequently, because of poor adherence to antihypertensive treatment, approximately $75 \%$ of patients with a diagnosis of hypertension do not achieve optimum blood-pressure control. ${ }^{[39]}$

\section{CONCLUSION}

Adherence to antihypertensive medications is a key to control blood pressure levels. Non adherence is the most significant cause of uncontrolled BP. Common barriers to adherence are under the patient's control, so that attention to them is a necessary and important step in improving adherence. The factors driving patients' 
adherence to medication are many but can be grouped under five main domains including socioeconomic factors, healthcare system related factors, disease related factors, therapy-related factors and patientrelated factors, identifying factors that affect medication adherence is the first step towards improving adherence

\section{REFERENCES}

1. Mekonnen HS, Gebrie MH, Eyasu KH, et al. Drug adherence for antihypertensive medications and its determinants among adult hypertensive patients attending in chronic clinics of referral hospitals in Northwest Ethiopia. BMC Pharmacology and Toxicology.2017;18(1):1-10

2. Gupta S, Dhamija JP, Mohan I, et al. Qualitative Study of Barriers to Adherence to Antihypertensive Medication among Rural Women in India. International Journal of Hypertension.2019

3. Agbor VN, Takah NF, Aminde LN. Prevalence and factors associated with medication adherence among patients with hypertension in sub-Saharan Africa:protocol for a systematic review and metaanalysis. BMJ Open. 2018;8(3).

4. Naghavi S, Mehrolhassani MH, Nakhaee $\mathrm{N}$, et al. Effective factors in non-compliance with therapeutic orders of specialists in outpatient clinics in Iran: a qualitative study. BMC Health Serv Res. 2019

5. Zelko E, Klemenc-Ketis Z, Tusek-Bunc K. medication adherence in elderly with polypharmacy living at home: a systematic review of existing studies. Mater sociomed. 2016;28(2):129-132.

6. Costa E, Giardini A, Savin M, et al. Interventional tools to improve medication adherence:review of literature.Patient Prefer Adherence. 2015;14:1303-14.

7. Jeannette Y. Wick. Adherence Issues in Elderly Patients. Pharmacy times. 2011.

8. Chudiak A, Uchmanowicz I, Mazur G. Relation between cognitive impairment and treatment adherence in elderly hypertensive patients. ClinInterv Aging. 2018;13:14091418.

9. Jin HK, Kim YH, Rhie SJ. Factors affecting medication adherence in elderly people. Dovepress.2016;10:2117-2125.

10. Okuno J, Yanagi H, Tomura S, et al. Compliance and medication knowledge among elderly Japanese home-care recipients. European Journal of Clinical pharmacology. 1999;55:145-149.

11. Trivedi RB, Ayotte B, Edelman D, et al. The association of emotional well-being and marital status with treatment adherence among patients with hypertension. J Behav Med. 2008;31(6):489-497.

12. Jia-Rong Wu, Lennie TA, Chung ML, et al. Medication Adherence Mediates the Relationship between Marital Status and Cardiac Event-Free Survival in Patients with Heart Failure. Heart Lung. 2012;41(2):107-114.

13. Manteuffel M, Williams S, Chen W, et al. Influence of Patient Sex and Gender on Medication Use, Adherence, and Prescribing Alignment with Guidelines. Journal of Women's Health. 2014;23(2).

14. Sundbom LT, Bingefors K. Women and men report different behaviours in, and reasons for medication non-adherence:a nationwide Swedish survey. Pharm Pract (Granada). 2012;10(4):207-221.

15. Braverman J, Dedier J. Predictors of medication adherence for African American patients diagnosed with hypertension. Ethnicity\& disease. 2009;19(4):396-400.

16. Kumaraswamy RC, Kauser MM, Jagadeesh MK, et al. Study of determinants of nonadherence to anti-hypertensive medications in essential hypertension at a Teaching Hospital in Southern India. Chrismed journal of health and research. 2015;2(1):57-60.

17. Shi S, Shen Z, Duan Y, et al. Association Between Medication Literacy and Medication Adherence Among Patients With Hypertension. Front Pharmacol. 2019;10:822.

18. Atinga RA, Yarney L, Gavu NM. Factors influencing long-term medication nonadherence among diabetes and hypertensive patients in Ghana:A qualitative investigation. PLoS ONE. 2018;13(3).

19. Alhewiti A. Adherence to Long-Term Therapies and Beliefs about Medications. Int J Family Med. 2014;2014:479596.

20. Horne R, Graupner L, Frost S, et al. Medicine in a multi-cultural society: the effect of cultural background on beliefs about medications. SocSci Med. 2004; 59(6):1307-13. 
21. Kalogianni A. Factors affect in patient adherence to medication regimen. Health Science Journal. 2011; 5(3):157-158.

22. Gu L, Wu S, Zhao S, et al. Association of Social Support and Medication Adherence in Chinese Patients with Type 2 Diabetes Mellitus. Int J Environ Res Public Health. 2017;14(12):1522.

23. Svensson S, Kjellgren KI, Ahlner J, et al. Reasons for adherence with antihypertensive medication. International journal of cardiology. 2000;76(2-3):157-63.

24. Kleinsinger F. The Unmet Challenge of Medication Nonadherence. Perm J. 2018; 22(1):18-033.

25. Polinski JM, Kesselheim AS, Frolkis JP, et al. A matter of trust: patient barriers to primary medication adherence. Health Educ Res. 2014;29(5):755-63.

26. Susan morse. High price of drugs is biggest issue in prescription adherence, physicians say.Healthcare finance. 2019.

27. Heath S. 5 Ways to Improve Medication Adherence in Chronic Care Patients. PATIENT CARE ACCESS NEWS. 2017.

28. Park HY, Seo SA, Yoo H, et al. Patient Prefer Adherence. 2018;12:175-181.

29. Huang J , Jiang Z, Zhang T, et al. Which Matters More for Medication Adherence Among Disabled People in Shanghai, China: Family Support or Primary Health Care? The Journal of Health Care Organization, Provision, and Financing. 2019;56:1-10.

30. Grodensky, CA, Golin, CE, Ochtera RD, et al. Systematic review: Effect of alcohol intake on adherence to outpatient medication regimens for chronic diseases. Journal of Studies on Alcohol and Drugs. 2012;73(6):899-910.

31. Bryson CL, Au DH, Sun H, et al. Alcohol screening scores and medication nonadherence. Annals of internal medicine. 2008;149(11):795-803.
32. Gonzalez A, Mimiaga MJ, Israel J, et al. Substance use predictors of poor medication adherence:the role of substance use coping among HIV-infected patients in opioid dependence treatment. AIDS Behav. 2013; 17(1):168-173.

33. Ashoorkhani M, Majdzadeh R, Gholami J,et al. Understanding Non-Adherence to Treatment in Hypertension: A Qualitative Study. Int J Community Based Nurs Midwifery. 2018;6(4):314-323.

34. Priyanka T, Lekhanth A, Revanth A, et al. Effect of Polypharmacy on Medication Adherence in Patients with Type 2 Diabetes mellitus. Indian Journal Of Pharmacy Practice. 2015;8(3):126-132.

35. Mukete BN, Ferdinand KC. Polypharmacy in Older Adults With Hypertension: A Comprehensive Review. Journal of Clinical Hypertension. 2016;18 (1):10-8

36. Ekor M. The growing use of herbal medicines: issues relating to adverse reactions and challenges in monitoring safety. Front Pharmacol. 2014;4:177.

37. Abegaz TM, Shehab A, Gebreyohannes EA, et al. Nonadherence to antihypertensive drugs: a systematic review and metaanalysis. Medicine. 2017;96(4).

38. Tedla YG, Bautista LE. Drug Side Effect Symptoms and Adherence to Antihypertensive Medication. American Journal of Hypertension. 2016;29(6) 772779 .

39. Vrijens B, Vincze GB, Urquhart J, et al. Adherence to prescribed antihypertensive drug treatments: longitudinal study of electronically compiled dosing histories. BMJ Research. 2008

How to cite this article: Satish S, Jose M, Shabaraya AR. Adherence to antihypertensive medications and its determinants in hypertensive patients - a complete review. International Journal of Research and Review. 2021; 8(2): 42-48. 